\title{
Planned Notification Study Accrual Threshold Percent
}

National Cancer Institute

\section{Source}

National Cancer Institute. Planned Notification Study Accrual Threshold Percent. NCI

Thesaurus. Code C94013.

An integer specifying a percentage of targ et accrual for a study, which when reached triggers a notification. 